\title{
Use of Syndromic Data for Enhanced Surveillance: MERS Like-Syndrome
}

\author{
Achintya N. Dey*1, Matthew Miller ${ }^{2}$, Michael Coletta ${ }^{1}$ and Umed Ajani ${ }^{1}$ \\ 'DHIS, CDC, Atlanta, GA, USA; ${ }^{2}$ McKing Consulting, Atlanta, GA, USA
}

\section{Objective}

To identify and monitor Middle East Respiratory Syndrome (MERS) like syndromes cases in the syndromic surveillance system.

\section{Introduction}

Centers for Disease Control and Prevention's (CDC) BioSense system receives near real-time health care utilization data from number of sources, including DoD and VA outpatient facilities, and nonfederal hospital EDs in the US to support all-hazards surveillance and situational awareness. However, the BioSense system lacks some critical functions such as creating ad hoc definition of syndrome or ad hoc query tool development. This limits CDC Emergency Operations Center's (EOC) ability to monitor new health events such as MERS - a viral respiratory illness first reported in Saudi Arabia in 2012. In May 2014, CDC confirmed two unlinked imported cases of MERS in the US - one in Indiana, the other in Florida. Upon report of a MERS case in Indiana, staff initiated joint efforts with EOC and several affected jurisdictions to enhance the surveillance of MERS irrespective of jurisdictions' preferred surveillance system.

\section{Methods}

In consultation with the state and local jurisdictions, five case definitions were developed to monitor MERS like syndromes: (1) was designed to search chief complaint: any mention of MERS or any mention of $\mathrm{CoV}$ or any mention of Oman or any mention of Camel or MiddleEast or Arabian or AbuDhabi or Egypt or Jordan or Kuwait or Qatar or UAE or United Arab Emirates or Bahrain or Iraq or Iran or Israel or Lebanon or Palestin or Saudi or Syria or Yemen; (2) was designed to focus solely on the travel terms: any mention of Oman or any mention of MiddleEast or Arabian or AbuDhabi or Egypt or Jordan or Kuwait or Qatar or UAE or United Arab Emirates or Bahrain or Iraq or Iran or Israel or Lebanon or Palestin or Saudi or Syria or Yemen; (3) was meant to be more specific, requiring the mention of both MERS and Travel: Part A search term included (MERS and Travel): any mention of MERS and any mention of Oman or any mention of Camel or MiddleEast or Arabian or AbuDhabi or Egypt or Jordan or Kuwait or Qatar or UAE or United Arab Emirates or Bahrain or Iraq or Iran or Israel or Lebanon or Palestin or Saudi or Syria or Yemen) or Part B search term included (CoV AND Travel): any mention of $\mathrm{CoV}$ and any mention of Oman or any mention of Camel or MiddleEast or Arabian or AbuDhabi or Egypt or Jordan or Kuwait or Qatar or UAE or United Arab Emirates or Bahrain or Iraq or Iran or Israel or Lebanon or Palestin or Saudi or Syria or Yemen; (4) was focused on secondary spread: any mention of Fever and Pneumonia or any mention of Fever and Acute Respiratory Distress; (5) used ICD-9-CM, and ICD-10-CM: 079.82, 480.3, V01.82, B34.2, B97.21, J12.81, U00-U49, U04.9.

These five case definitions were then operationalized in SQL, R-Script, SAS, and ESSENCE languages and sent out to each participating state and local jurisdictions to run the weekly query on their syndromic surveillance systems for sharing the results with the CDC. BioSense staff compiled the results and set up a weekly reporting system on MERS like syndrome cases to EOC.

\section{Results}

From May through July, 2014, fifteen reporting jurisdictions participated in MERS enhanced surveillance. These 15 reporting jurisdictions reported from 822 civilian hospitals ED facilities. BioSense staff created weekly reports for the CDC's EOC. Reports included five case definitions and a table reporting jurisdiction name, date, number of MERS case investigated and number of MERS cases ruled out. During this enhanced surveillance time period 171 probable MERS cases were identified and all of them were ruled out.

\section{Conclusions}

In spite of limitations such as low participation and lack of agreement among the jurisdictions in sharing data, CDC BioSense staff was able to provide meaning full data to EOC for enhanced surveillance on MERS like syndrome. Similar collaborative efforts between BioSense programs, CDC subject matter experts and jurisdictions will help develop more comprehensive definitions to conduct enhanced surveillance at the national level using multiple syndromic surveillance systems.

\section{Keywords}

MERS; syndromic surveillance; ED facilities

\section{Acknowledgments}

Participating State and Jurisdictions

*Achintya N. Dey

E-mail: aad2@cdc.gov 